\title{
Captación electroquímica de dióxido de carbono como opción para mitigar el cam- bio climático, presentado en el 6to Seminario internacional de expertos en trata- miento de efluentes industriales y residuos
}

\author{
SANDRA PAOLA GUTIÉRREZ MONTALVÁN', RONNY FLORES 1* \\ 'Laboratorio de Química Ambiental y Sostenible, \\ Facultad de Ciencia Químicas de la Universidad Central del Ecuador. \\ Francisco Viteri y Gilberto Sobral s/n, Ciudad Universitaria, Quito. \\ Apartado 17-03-7369, Ecuador. Tel.: (593-02) 2502-262, 2502-456 \\ *Correspondencia: raflores@uce.edu.ec
}

Recibido: 25 de octubre de 2015, Aceptado: 8 de septiembre de 2016

\section{Resumen}

Este artículo es parte del bto Seminario internacional de expertos en tratamiento de efluentes industriales y residuos. Se diseñó una celda para la captura electroquímica de dióxido de carbono disuelto en agua de mar artificial mediante el fenómeno de electrodeposición mineral (precipitación del $\mathrm{CO}_{2}$ como carbonato de calcio y magnesio) para mitigar el efecto invernadero. La celda electroquímica está constituida por una malla cilíndrica de acero inoxidable como cátodo y una barra de grafito como ánodo. La celda presentó una adecuada precipitación de los carbonatos, un desgaste mínimo de los electrodos y un gasto energético pequeño. En el agua de mar artificial se realizaron mediciones de concentración de dióxido de carbono disuelto, con un electrodo selectivo, y de concentración de calcio y magnesio, por absorción atómica, para determinar la cantidad de carbonatos precipitados mientras se variaba la intensidad de corriente y tiempo de aplicación. Se encontró que el mayor porcentaje de captura de $\mathrm{CO}_{2}(99.17 \%$ ) se obtuvo cuando se utilizaron dos amperios durante 60 minutos. El mejor sistema electrolítico se aplicó a una muestra de agua de mar natural de la Antártida para verificar su eficacia, lográndose un alto porcentaje de captura de dióxido de carbono (99.19\%). Concluyéndose que el método desarrollado es eficiente, factible de implementar $y$, sobre todo, que puede ayudar en la mitigación del efecto invernadero.

Palabras clave: Electrodeposición mineral, captura electroquímica, dióxido de carbono, efecto invernadero.

\section{Electrochemical capturing of carbon dioxide as choice to mitigate climate chan- ge" at 6th International seminar of experts on the} treatment of industrial effluents and residues

\begin{abstract}
This article is part of the 6th International seminar of experts on the treatment of industrial effluents and residues. An electrochemical cell to capture the carbon dioxide dissolved in artificial seawater was designed by mineral electrodeposition phenomenon (precipitation of $\mathrm{CO}_{2}$ like calcium carbonate and magnesium) to mitigate the greenhouse effect. A cylindrical stainless steel mesh as cathode and a graphite rod as anode formed the electrochemical cell. The cell showed an appropriate precipitation of carbonates, a minimal wear of the electrodes and small energy consumption. Measurements of dissolved carbon dioxide were performed in the artificial seawater with a selective electrode and concentration of calcium and magnesium by atomic absorption to determinate the amount of precipitated carbonates while it was changing the amperage and time of application. The highest percentage of capture of $\mathrm{CO}_{2}(99.17 \%)$ was obtained when two amperes per 60 minutes were used. The best electrolytic system was applied in a sample of natural seawater from Antarctica, and it got a high percentage of capture of carbon dioxide (99.19\%). It can be concluded that the developed method is efficient, feasible to implement and the most important thing, it can help mitigating the greenhouse effect.
\end{abstract}

Key words: Mineral electrodeposition, electrochemical capturing, carbon dioxide, greenhouse effect. 


\section{Introducción}

El dióxido de carbono $\left(\mathrm{CO}_{2}\right)$ es un gas de efecto invernadero que se encuentra naturalmente en la atmósfera, pero las actividades humanas han aumentado significativamente su concentración en la atmósfera contribuyendo así al calentamiento global del planeta. La principal fuente de emisión de dióxido de carbono a la atmósfera es la quema de combustibles fósiles (gas natural, petróleo) y biomasa (leña) en procesos industriales, transporte y actividades domiciliarias (cocina y calefacción). Los incendios forestales y de pastizales constituyen también una fuente importante de $\mathrm{CO}_{2}$ atmosférico. Los esfuerzos para reducir su concentración en la atmósfera se resumen en cuatro grandes políticas: Disminución en el uso de combustibles fósiles; Incremento en el uso de energías renovables; Incremento en la eficiencia energética y Captura y almacenamiento de dióxido de carbono (Aguinaco, 2008; Herzog et al., 2000). El presente trabajo de investigación se enfoca en la cuarta políitica, captura y almacenamiento de $\mathrm{CO}_{2}$, para mitigar el calentamiento global.

\section{Captura y almacenamiento de dióxido de carbono}

La captura y almacenamiento consiste en el secuestro del $\mathrm{CO}_{2}$ antropogénico y confinarlo en un reservorio donde no pueda causar daño. La aplicación de tecnologías de captura y almacenamiento de $\mathrm{CO}_{2}$ pueden reducir las emisiones de dióxido de carbono a la atmósfera hasta en un $\mathbf{9 0}$ por ciento en el sector industrial y energético. Desde hace mucho se vienen utilizando técnicas para separar y capturar rutinariamente el $\mathrm{CO}_{2}$ como subproducto de procesos industriales. Por ejemplo, en la producción sintética de amoníaco, la producción de hidrógeno, la calcinación de la piedra caliza, la captura en el agua para dar efervescencia a las bebidas carbonatadas, o su empleo como agente extractante en la obtención de la cafeína. Sin embargo, son procesos muy diferentes a los de captura y almacenamiento en los sectores puntuales de generación eléctrica, transporte e industria para intentar atenuar los efectos del cambio climático (Herzog et al., 2000).

Existen opciones para capturar el dióxido de carbono producido en las centrales eléctricas o plantas industriales, que luego se almacena, por largos períodos, ya sea en formaciones geológicas del subsuelo, en el océano o bajo otras condiciones. Son tecnologías con muchas cuestiones por resolver desde el punto de vista ambiental y económico. En la actualidad no existe un método de captura del dióxido de carbono eficiente y de bajo costo que sirva para mitigar el efecto invernadero.

La tecnología mejor estudiada es el almacenamiento geológico de $\mathrm{CO}_{2}$. Primero se realiza la captura, de tal forma que pueda disponerse de $\mathrm{CO}_{2}$ con la mayor pureza posible, para su posterior inyección. Las fuentes existentes no son rentables debido a la gran cantidad de aire que se añade a la combustión, las emisiones de $\mathrm{CO}_{2}$ aparecen muy diluidas en nitrógeno. Por ejemplo, en el caso de las emisiones de las centrales eléctricas de carbón, el $\mathrm{CO}_{2}$ supone únicamente un $10-12 \%$ en volumen y para conseguir un secuestro eficaz, en estos gases de escape, el $\mathrm{CO}_{2}$ deber ser separado y concentrado (Aguinaco, 2008). La selección del lugar de almacenamiento requiere un estudio detallado de numerosos parámetros que definen la idoneidad o no del sitio. Los sitios de almacenamiento pueden clasificarse en: Formaciones geológicas (yacimientos de petróleo agotados, yacimientos de gas exhaustos, capas de carbón no explotables con posibilidades de recuperación de metano, formaciones profundas con agua salada, formaciones de pizarras bituminosas con altos contenidos en materia orgánica, áreas geotérmicas de baja entalpía, yacimientos salinos susceptibles de disolución), en las masas de aguas profundas o en forma de minerales carbonatados. En el caso del almacenamiento oceánico profundo, existe el riesgo de aumentar enormemente el problema de la acidificación de los océanos, un problema que se origina también del exceso de dióxido de carbono presente ya en la atmósfera. La adición de $\mathrm{CO}_{2}$ al océano o la formación de depósitos de $\mathrm{CO}_{2}$ líquido en el fondo oceánico a escala industrial alteraría el medio químico local. Los experimentos han demostrado que las altas concentraciones sostenidas de $\mathrm{CO}_{2}$ causarían mortalidad entre los organismos oceánicos. Los efectos del $\mathrm{CO}_{2}$ en los organismos marinos tendrían repercusiones en el ecosistema. Los efectos crónicos de la inyección directa de $\mathrm{CO}_{2}$ en el océano sobre ecosistemas de amplias zonas oceánicas y a largo plazo aún no han sido estudiados (Metz et al., 2005). Las formaciones geológicas son consideradas actualmente los sitios más plausibles de secuestro de carbono (Aguinaco, 2008).

En la carbonatación mineral para la fijación de carbono, el $\mathrm{CO}_{2}$ se convierte en carbonatos inorgánicos sólidos mediante reacciones químicas con óxidos alcalinos y alcalinotérreos, como el óxido de magnesio 
$(\mathrm{MgO})$ y el óxido de calcio ( $\mathrm{CaO})$, que están presentes en las rocas de silicatos de formación natural como la serpentina y el olivino. Las reacciones químicas entre estos materiales y el $\mathrm{CO}_{2}$ producen compuestos como el carbonato de magnesio $\left(\mathrm{MgCO}_{3}\right)$ y el carbonato de calcio $\left(\mathrm{CaCO}_{3}\right.$, comúnmente conocido como piedra caliza). La cantidad de óxidos metálicos presentes en las rocas de silicatos que pueden encontrarse en la corteza terrestre excede de las cantidades necesarias para fijar todo el $\mathrm{CO}_{2}$ que produciría la combustión de todas las reservas de combustibles fósiles existentes. El proceso de carbonatación mineral se produce de forma natural y se conoce como "meteorización". En la naturaleza, el proceso es muy lento, por lo que debe ser acelerado de forma considerable a fin de convertirlo en un método de almacenamiento viable para el $\mathrm{CO}_{2}$ captado procedente de fuentes antropogénicas. El proceso de carbonatación mineral requeriría la explotación de entre 1.6 y 3.7 toneladas de silicatos por cada tonelada de $\mathrm{CO}_{2}$, y produciría de 2.6 a 4.7 toneladas de materiales desechables por cada tonelada de $\mathrm{CO}_{2}$ almacenado como carbonatos. Por tanto, se trataría de una operación de grandes dimensiones, con un impacto ambiental similar al de las actuales actividades de explotación minera a cielo abierto y a gran escala. La serpentina también suele contener crisotilo, que es una forma natural de asbesto, por lo que su presencia exige medidas de vigilancia y mitigación de la misma índole que las existentes en el sector de la minería. La medida en que puede utilizarse la carbonatación mineral no puede determinarse en este momento, se halla en la fase de investigación, ya que depende de la cantidad desconocida de reservas de silicato que pueden ser técnicamente explotadas y de cuestiones ambientales como las que se han señalado supra (Metz et al, 2005).

\section{Disolución marina de $\mathrm{CO}_{2}$}

Casi todo el carbono disuelto en el mar está en forma de iones: un $85 \%$ en forma de bicarbonato, $\mathrm{HCO}_{3}$, y un $15 \%$ en forma de carbonato, $\mathrm{CO}_{3}{ }^{2}$. Tan sólo el 0.5 $\%$ de todo el carbono inorgánico disuelto en el mar está en forma de $\mathrm{CO}_{2}$ gaseoso, y la concentración de ácido carbónico $\mathrm{H}_{2} \mathrm{CO}_{3}$ es aún menor. El mar contiene en disolución, aproximadamente, 50 veces más carbono que el $\mathrm{CO}_{2}$ del aire: $40,000 \mathrm{PgC}$ (petagramos de carbono) y $750 \mathrm{PgC}$, respectivamente. Entre su superficie y el aire existe, en ambos sentidos, un continuo transvase de $\mathrm{CO}_{2}$. En algunas épocas el mar actúa como fuente de $\mathrm{CO}_{2}$ atmosférico, y en otras como sumidero (Hansell \& Carlson, 2001). El intercambio de $\mathrm{CO}_{2}$ en- tre el mar y la atmósfera depende directamente de la pequeña fracción del $\mathrm{CO}_{2}$ presente en la superficie del mar. Su presión en la superficie del agua $\left(\mathrm{pCO}_{2}\right)$ se mantiene en equilibrio con su presión parcial en la atmósfera, existiendo un continuo flujo molecular aire-mar/mar-aire, que regula y hace variar las concentraciones de carbono en ambos reservorios. Si por algún motivo, disminuye la presión $\mathrm{pCO}_{2}$ del agua, se produce un pase neto de moléculas de $\mathrm{CO}_{2}$ del aire al mar. Esto es, el océano absorbe $\mathrm{CO}_{2}$ del aire, y aumenta la $\mathrm{pCO}_{2}$ del agua de nuevo, y viceversa. La $\mathrm{pCO}_{2}$ del agua depende directamente de su concentración $\left[\mathrm{CO}_{2}\right]$ (moles de $\mathrm{CO}_{2}$ por kg de solución) e inversamente de su solubilidad. Por ejemplo, cuando el agua se enfría, la solubilidad del $\mathrm{CO}_{2}$ aumenta (pues los gases son más solubles en agua fría que caliente), con lo que disminuye la presión $\mathrm{pCO}_{2}$ del agua. Se produce un desequilibrio entre las presiones $\mathrm{pCO}_{2}$ de la superficie del agua y del aire en contacto con ella, y entonces el agua absorbe más $\mathrm{CO}_{2}$ aumentando su concentración $\left[\mathrm{CO}_{2}\right]$. Como consecuencia, disminuye la concentración $\left[\mathrm{CO}_{2}\right]$ del aire. El proceso inverso ocurre cuando el agua se calienta. Por lo tanto, en principio, en las épocas de enfriamiento de las aguas, el océano absorbe $\mathrm{CO}_{2}$ del aire y hace disminuir su concentración en la atmósfera, y, por el contrario, en las épocas en que las aguas se calientan, el océano suelta $\mathrm{CO}_{2}$ y aumenta su concentración atmosférica (También se esperaría que en las aguas frías de la Antártida la disolución del $\mathrm{CO}_{2}$ sea mayor.) (Hansell \& Carlson, 2001). Este comportamiento puede describirse mediante la ley de Henry que enuncia que, a una temperatura constante, la cantidad de gas disuelto en un líquido es directamente proporcional a la presión parcial que ejerce ese gas sobre el líquido. En la atmósfera, el dióxido de carbono, se encuentra en una concentración de unos 400 ppm. Como consecuencia del bajo nivel de $\mathrm{CO}_{2}$ atmosférico, el agua en equilibrio con la atmósfera, totalmente libre de alcalinidad (capacidad de neutralizar $\mathrm{H}^{+}$), contiene solo un nivel muy bajo de dióxido de carbono, del orden de $10^{-5}$ moles/litro. Sin embargo, la formación de $\mathrm{HCO}_{3}$ y $\mathrm{CO}_{3}{ }^{2 \cdot}$ incrementa grandemente la solubilidad del dióxido de carbono (Manahan, 2007).

\section{Electrodeposición de minerales en agua de mar}

Existe una relación directa entre la concentración del dióxido de carbono que se encuentra en la atmósfera y la que se encuentra en los océanos. Este hecho permite plantear una captura del $\mathrm{CO}_{2}$ en el agua para 
disminuir la concentración de $\mathrm{CO}_{2}$ en la atmósfera, que sería un método alternativo de captura de $\mathrm{CO}_{2}$. El método consiste en capturar el $\mathrm{CO}_{2}$, producido por la industria, mediante un proceso llamado electrodeposición mineral. El $\mathrm{CO}_{2}$ se transforma en una forma sólida a partir de una serie de reacciones químicas con elementos que se encuentran de forma natural en el agua de mar, principalmente el calcio y magnesio. Este proceso es muy lento pero puede acelerarse aplicando una intensidad de corriente mediante una celda electrolítica.

El agua de mar contiene nueve elementos principales: sodio, magnesio, calcio, potasio, estroncio, cloro, azufre, bromo, y carbono. Estos elementos comprenden más de $99.9 \%$ del total de las sales disueltas en el océano. Al aplicar una corriente eléctrica directa entre electrodos y un electrolito como el agua de mar, en el cátodo se da la precipitación de carbonatos de calcio y magnesio e hidróxido de magnesio y el desprendimiento de hidrógeno, mientras que en el ánodo se produce oxígeno y cloro. Un modelo cualitativo de los procesos electroquímicos del acrecentamiento de minerales se propone en la Figura 1, donde se observa la formación de carbonato y todas las posibles reacciones electroquímicas en el agua de mar (Hilbertz, 1979).

También, se ha propuesto la reducción electroquímica del $\mathrm{CO}_{2}$ para la obtención de hidrocarburos y $\mathrm{CO}$ en sistemas acuosos y no acuosos. El mecanismo de la reducción electroquímica de $\mathrm{CO}_{2}$ no ha sido, todavía, elucidado satisfactoriamente (Jitaru, 2007, Levitan 2013).

\section{Parte experimental}

\section{Reactor electrolítico}

En un estudio previo se determinó que el mejor sistema de electrodos para la electrodeposición es el conformado por malla de acero como cátodo y grafito como ánodo. Los electrodos son inertes y no forman precipitados que podrían interferir con los ensayos. Como ánodo se utilizaron los núcleos de grafito de las pilas secas tipo D. Como cátodo malla de acero inoxidable ASTM mesh 20 grado AISI 304, resistente a la corrosión.

Se construyó un reactor electrolítico de vidrio Pyrex con tubuladura de desprendimiento para liberación de gases, tapa de esmeril plano para cierre hermético con puertos para la introducción de los electrodos, medidor de $\mathrm{pH}$, conductividad y temperatura tal como se muestra en la Figura 2. A la malla de acero de 4x10 $\mathrm{cm}$ se le dio forma cilíndrica y la varilla de grafito se ubicó en el centro.

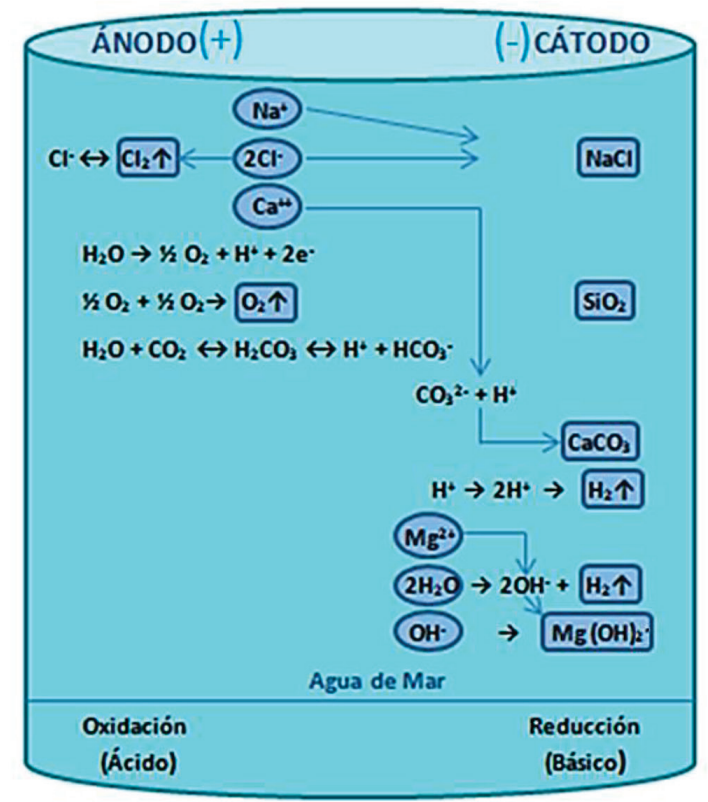

Figura 1. Reacciones electroquímicas en agua de mar. Modificado de Hilbertz (1979) 


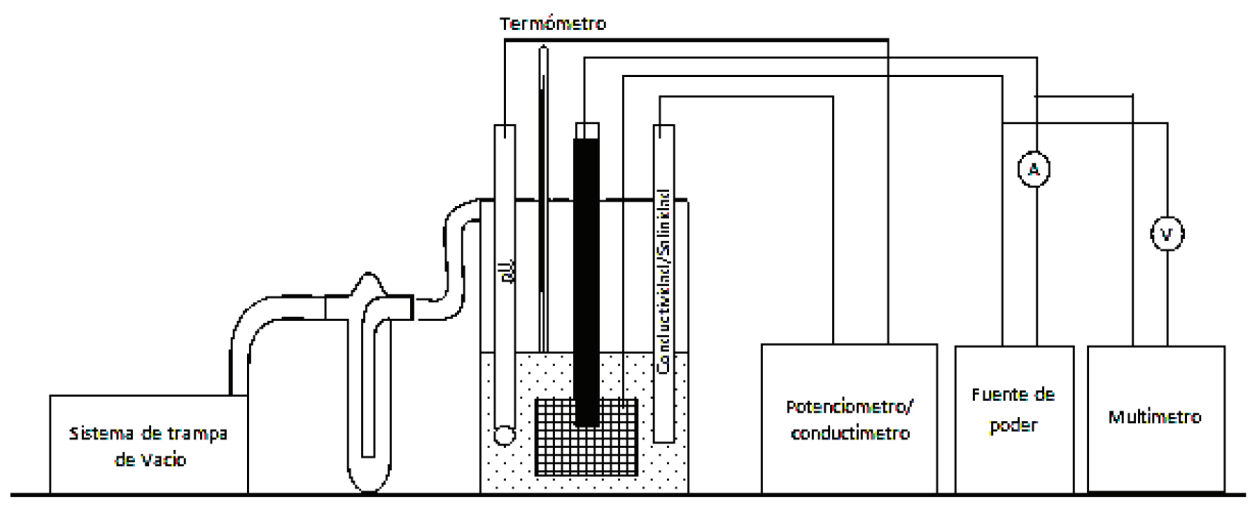

Figura 2. Diagrama de reactor electrolítico

Se prepararon cuatro litros de agua de mar artificial mezclando las sales descritas en la Tabla 1 con reactivos grado p.a. y agua tipo $\mathrm{I}$.

Tabla 1. Principales sales del agua de mar

\section{Captura electroquímica de $\mathrm{CO}_{2}$}

Se colocó exactamente $100 \mathrm{~mL}$ de agua de mar artificial en la celda del reactor y se ubicaron adecua-

\section{COMPONENTE CANTIDAD, (g/L)}

Cloruro de sodio

24

Cloruro de magnesio

Sulfato neutro de sodio

4

Cloruro de calcio

Cloruro de potasio

Bicarbonato de sodio

Bromuro de sodio

Ácido bórico

Cloruro de estroncio

Fluoruro de sodio

0.003

Tomado de Pontes (2011). damente el par de electrodos, previamente pesados, dentro de la celda, así como todos los medidores de parámetros. Se moduló la fuente de poder con amperaje regulable, marca VED TECHNISCH, a los valores de intensidad de corriente determinados $(0.5,1$ y 2 A) y se medió la diferencia de potencial de la celda mediante un multímetro marca PROSKIT. Se aplicó la intensidad de corriente por un tiempo especificado $(15,30$ y $60 \mathrm{~min})$. Durante todo el proceso se mantuvo agitación magnética a velocidad constante. Se verificó la temperatura con un termómetro digital marca FISHER $\left(A \pm 0.1^{\circ} \mathrm{C}\right)$. Finalizado el tiempo de reacción se dejó reposar por 5 minutos y se procedió a retirar los electrodos que se secaron, pesaron y guardaron adecuadamente. La solución electrolítica se filtró en un papel cuantitativo previamente pesado. Se almacenaron adecuadamente los filtrados y precipitados para posteriores análisis. Se facilitó la salida de los gases que se desprendieron durante la reacción con una trompa de agua que genera vacío y se recolectaron en una trampa conteniendo una solución $1 \mathrm{M}$ de $\mathrm{NaOH}$. Las determinaciones se realizaron por triplicado.

\section{Medición de parámetros}

Se cuantificó la cantidad de $\mathrm{CO}_{2}$ disuelto en el agua de mar artificial con un electrodo selectivo de gas 
$\mathrm{CO}_{2}$, según manual de operación del equipo. Se utilizó un potenciómetro con electrodo de $\mathrm{Ag} / \mathrm{AgCl}$ para la determinación del $\mathrm{pH}$ y un conductímetro para la medición de la conductividad y salinidad, de acuerdo al manual de los equipos, todos marca METTLER TOLEDO. Estos parámetros fueron evaluados en las soluciones iniciales y finales después de haber realizado el proceso de captura electrolítica.

Para deducir la composición del sólido formado se pesó el precipitado producido y en el filtrado de la solución electrolítica se analizó calcio y magnesio. Se utilizó el método estándar APHA 311 .D con un equipo de absorción atómica con lámpara de cátodo hueco para calcio y magnesio marca PERKIN ELMER. Las mediciones se realizaron en las soluciones iniciales y finales después de haber efectuado el proceso de captura electrolítica.

Con los datos obtenidos se realizó un análisis de varianza para un diseño factorial $A x B$ de tres niveles con tres repeticiones, para su elaboración se utilizó una hoja de cálculo de Excel 2010. La variable de respuesta fue la concentración de dióxido de carbono capturado en términos de porcentaje.

\section{Efectividad del método}

Para establecer la efectividad de método se realizó una captura electroquímica de $\mathrm{CO}_{2}$ en $100 \mathrm{~mL}$ de agua de mar natural, siguiendo el mismo procedimiento que para el agua artificial. Se utilizó el mejor sistema electroquímico encontrado para el agua artificial.

\section{Resultados y discusión}

En la Tabla 2 se muestran los parámetros medidos en el agua de mar sintética y en el agua de mar natural procedente de la Antártida (tomada frente a la Estación antártica ecuatoriana Pedro Vicente Maldonado, verano Austral 2013, que fue transportada y conservada en refrigeración a $4^{\circ} \mathrm{C}$ hasta el momento de los análisis).

Tabla 2. Parámetros iniciales de las muestras a $22^{\circ} \mathrm{C}$ y $0.78 \mathrm{~atm}$

\begin{tabular}{lcc}
\hline \multicolumn{1}{c}{ PARÁMETRO } & $\begin{array}{c}\text { AGUA DE MAR } \\
\text { SINTÉtICA }\end{array}$ & $\begin{array}{c}\text { AGUA DE MAR } \\
\text { NATURAL } \\
\text { DE LA ANTÁRTIDA }\end{array}$ \\
\hline $\mathrm{pH}$ & 8.04 & 7.05 \\
Conductividad $(\mathrm{mS} / \mathrm{cm})$ & 52.10 & 47.80 \\
Salinidad $(\mathrm{psu})^{1}$ & 34.84 & 31.40 \\
Concentración $\mathrm{Ca}(\mathrm{mg} / \mathrm{L})$ & 147.50 & 190.00 \\
Concentración $\mathrm{Mg}(\mathrm{mg} / \mathrm{L})$ & 1125.00 & 775.00 \\
Concentración inicial $\mathrm{CO}_{2}(\mathrm{mg} / \mathrm{L})$ & 282.08 & 292.78 \\
Concentración de carbonatos $(\mathrm{mg} / \mathrm{L})^{2}$ & 384.66 & 399.24 \\
\hline
\end{tabular}

${ }^{1}$ PSU (Practical Salinity Units), unidad de medida de la salinidad correspondiente a la relación entre la conductividad de una muestra de agua de mar y la de una solución estándar de $\mathrm{KCl}$ formado por 32,4356 gramos de sal disuelta en $1 \mathrm{~kg}$ de solución a $15^{\circ} \mathrm{C}$.

${ }^{2}$ Concentración de carbonatos según la reacción: $\mathrm{CO}_{2}+\mathrm{H}_{2} \mathrm{O}->\mathrm{CO}_{3}{ }^{2-}+2 \mathrm{H}^{+}$

El agua de mar artificial presentó valores diferentes a la muestra de agua de mar natural ya que no tiene algunas de las sales traza que están presentes en una muestra natural, sin embargo, las composiciones son comparables. Hay que tomar en cuenta, además, que las concentraciones de las especies presentes en el agua de mar dependen mucho del sitio donde se obtuvieron. 


\section{Selección del sistema electroquímico para la captura del $\mathrm{CO}_{2}$ disuelto en agua de mar}

El agua de mar es una matriz compleja con muchas sales en solución, por lo que para el estudio de este sistema se consideraron únicamente las sales de interés como son: $\mathrm{NaCl}, \mathrm{Ca}\left(\mathrm{HCO}_{3}\right)_{2}, \mathrm{MgCl}_{2}$ y $\mathrm{CaCl}_{2}$. Las principales reacciones que se producen en el seno de la solución y que permiten la electrodeposición mineral son (Hilbertz, 1979):

lonización de sales:

$$
\begin{aligned}
\mathrm{NaCl} & \rightarrow \mathrm{Na}^{+}+\mathrm{Cl}^{-} \\
\mathrm{CaCl}_{2} & \rightarrow \mathrm{Ca}^{2+}+2 \mathrm{Cl}^{-} \\
\mathrm{MgCl}_{2} & \rightarrow \mathrm{Mg}^{2+}+2 \mathrm{Cl}^{-} \\
\mathrm{Ca}\left(\mathrm{HCO}_{3}\right)_{2} & \rightarrow \mathrm{Ca}^{2+}+2 \mathrm{HCO}_{3}^{-}
\end{aligned}
$$

Reacciones de óxido - reducción:

$$
\begin{array}{ll}
2 \mathrm{Cl}^{-}-2 \mathrm{e}^{-} \Leftrightarrow \mathrm{Cl}_{2}(\mathrm{~g}) & -(+1.36 \mathrm{~V}) \text { ánodo } \\
2 \mathrm{H}_{2} \mathrm{O}+2 \mathrm{e}^{-} \Leftrightarrow \mathrm{H}_{2}(\mathrm{~g})+2 \mathrm{OH}^{-} & (-0.83 \mathrm{~V}) \text { cátodo }
\end{array}
$$

Productos:

$$
\begin{gathered}
\mathrm{Na}^{+}+\mathrm{OH}^{-} \rightarrow \mathrm{NaOH}(\mathrm{ac}) \\
\mathrm{Mg}^{2+}+2 \mathrm{OH}^{-} \rightarrow \mathrm{Mg}(\mathrm{OH})_{2}(\mathrm{~s}) \downarrow \\
\mathrm{HCO}_{3}^{-}+\mathrm{OH}^{-} \rightarrow \mathrm{CO}_{3}^{2-}+2 \mathrm{H}_{2} \mathrm{O} \\
\mathrm{Ca}^{2+}+\mathrm{CO}_{3}{ }^{2-} \rightarrow \mathrm{CaCO}_{3}(\mathrm{~s}) \downarrow \\
\mathrm{Mg}^{2+}+\mathrm{CO}_{3}{ }^{2-} \rightarrow \mathrm{MgCO}_{3}(\mathrm{~s}) \downarrow
\end{gathered}
$$

Por tanto, los productos finales son: $\mathrm{Cl}_{2}(\mathrm{~g}), \mathrm{H}_{2}(\mathrm{~g})$, que se desprenden como gases, $\mathrm{NaOH}$ (ac) en solución, que da el pH básico, y los precipitados $\mathrm{Mg}(\mathrm{OH})_{2}(\mathrm{~s})$, $\mathrm{CaCO}_{3}(\mathrm{~s})$ y $\mathrm{MgCO}_{3}(\mathrm{~s})$.

Se midió la concentración de $\mathrm{CO}_{2}$ antes y después del tratamiento y se calculó el porcentaje de captura (Figura 3). Además, se determinó la masa de precipitado adherida en la malla de acero, el desgaste del grafito, la masa de precipitado en el papel filtro. Se calculó la masa de calcio y magnesio precipitados como carbonatos y de magnesio como hidróxido lo que permitió deducir la composición del precipitado.

El análisis de varianza indicó que tanto el tiempo como la intensidad de corriente tienen una gran influencia en la captura del $\mathrm{CO}_{2}$ ya que existe diferencia significativa entre tratamientos con un $95 \%$ de confianza.

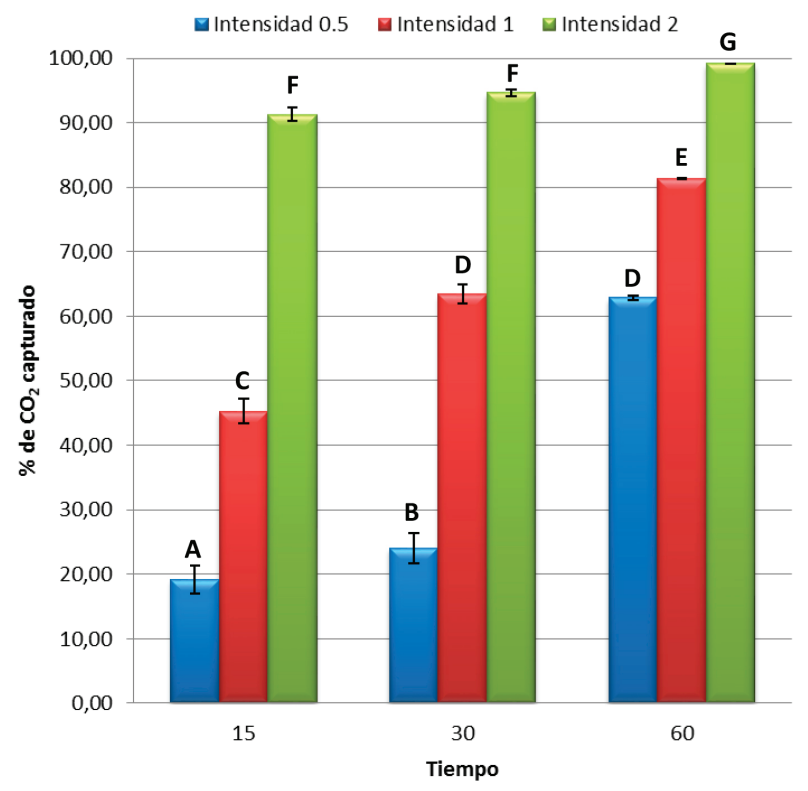

Figura 3. Porcentaje de $\mathrm{CO}_{2}$ capturado en función del tiempo (min.) y la intensidad de corriente (A). Letras iguales significa tratamientos estadísticamente iguales. Las barras de error corresponden a una desviación estándar de tres determinaciones

Se realizó una prueba de Tukey para comparar entre tratamientos. La prueba demostró que existen siete grupos de tratamientos que son estadísticamente diferentes con un nivel de significancia de 0.05. Los grupos $D$ y $\mathrm{F}$ están formados por dos tratamientos cada uno que son estadísticamente iguales, pues no existe entre ellos una diferencia significativa en el porcentaje de $\mathrm{CO}_{2}$ capturado. La mejor captura de $\mathrm{CO}_{2}$ se obtuvo con el tratamiento $G$, en el que se aplicó 2 A durante 60 min, con una remoción del $99 \%$. Este tratamiento fue el que se utilizó en la muestra de agua natural para determinar la efectividad del método. 


\section{Efectividad del método}

Para determinar la efectividad del método se procedió a realizar un ensayo en el que se aplicó el mejor sistema electrolítico encontrado (ensayo $G$ ) a una muestra de agua de mar natural de la Antártida (Figura 4).

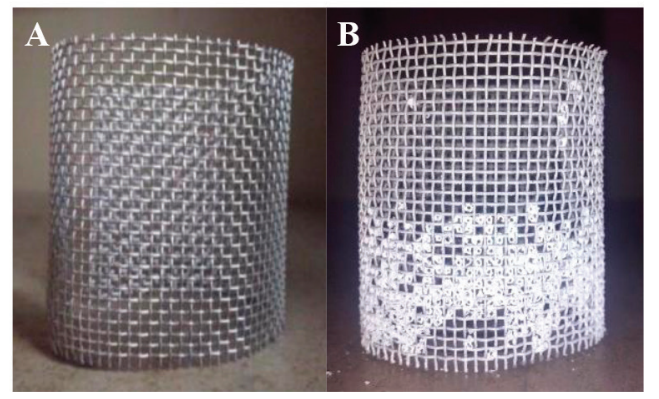

Figura 4. Electrodo de malla de acero $(A)$ antes y (B) después de la carbonatación en la muestra de agua de mar natural, donde puede observarse la acumulación de carbonatos en el electrodo

Se observó una importante capa de precipitado depositado sobre la malla (Figura 4), un desgaste mínimo de los electrodos y una salida eficiente de cloro. La masa total de precipitado fue de $234.4 \mathrm{mg}$ en los 100 $\mathrm{mL}$ de agua de mar natural, el $92.3 \%$ quedó adherido en la malla del electrodo, $6.0 \%$ corresponde a carbonato de calcio, $18.7 \%$ a carbonato de magnesio, $25.3 \%$ a hidróxido de magnesio y $50.0 \%$ a otros precipitados. Hubo un desgaste del $3.9 \%$ del electrodo de grafito. La concentración inicial de $\mathrm{CO}_{2}$ fue $292.78 \mathrm{mg} / \mathrm{L}$ y después de la carbonatación bajó a $2.38 \mathrm{mg} / \mathrm{L}$, que representa un $99.2 \%$ de remoción. En solución permanecieron $70.4 \%$ del ion calcio y $38.7 \%$ del ion magnesio lo que indica que puede continuarse con la carbonatación pues no se agotaron los iones que precipitan el carbonato.

\section{Conclusiones}

Para obtener capturas de $\mathrm{CO}_{2}$ mayores al $90 \%$ deben aplicarse intensidades de corriente de $2 \mathrm{~A}$, el tiempo mejora la deposición de carbonatos.

Debe considerarse que la captura electrolítica en mar abierto no sólo atraparía el $\mathrm{CO}_{2}$ proveniente de la disolución atmosférica; sino también el $\mathrm{CO}_{2}$ de los procesos biológicos y los carbonatos y bicarbonatos provenientes de la disolución de las formaciones rocosas dentro del océano, por lo que este proceso debería realizarse en un sistema aislado donde la única fuente de carbono antropogénico provenga del $\mathrm{CO}_{2}$ que se disuelve de la atmósfera o que se inyecta de la industria. Los modelos energéticos y económicos actuales indican que la principal contribución de un sistema de captación y almacenamiento de $\mathrm{CO}_{2}$ a la mitigación del cambio climático provendría de su uso en el sector de la generación de electricidad.

El presente trabajo de investigación plantea una alternativa a la captura geológica de $\mathrm{CO}_{2}$, que están tratando de implementar, actualmente, diferentes industrias para disminuir sus emisiones de este gas al ambiente. En la electrodeposición mineral en lugar de transportar e inyectar el $\mathrm{CO}_{2}$ de las industrias en formaciones geológicas o en aguas profundas, se lo capturaría mediante un burbujeo controlado, en agua de mar dentro de una celda similar a la descrita en esta investigación, pero con dimensiones industriales (para que sea viable se necesitará en el futuro cercano electricidad relativamente barata). El $\mathrm{CO}_{2}$ precipitaría en forma de sales de carbonatos de calcio y magnesio. El agua de mar tiene una alta concentración de calcio y magnesio que están disponibles para capturar el $\mathrm{CO}_{2}$ que se burbujee en el seno de la celda electroquímica. Adicionalmente, con este proceso se obtendrían sales muy apetecidas en el sector industrial como son los carbonatos, además de hidrógeno y cloro. Lo que demuestra esta investigación es que existen alternativas para disminuir las emisiones de $\mathrm{CO}_{2}$ a la atmósfera que deben ser analizadas con mayor profundidad.

Hay que considerar que ninguna opción tecnológica proporcionará por sí sola todas las reducciones de emisiones necesarias para lograr la estabilización del $\mathrm{CO}_{2}$, sino que se necesitará una serie de medidas de mitigación. La mayor parte de los escenarios prevén que el suministro de energía primaria seguirá estando dominado por los combustibles fósiles hasta, al menos, mediados de siglo. Las opciones tecnológicas conocidas podrían alcanzar un amplio margen de niveles de estabilización atmosférica, pero la puesta en marcha requeriría cambios socioeconómicos e institucionales (Metz et al, 2005).

\section{Agradecimientos}

Los autores agradecen a la Universidad Central del Ecuador y al Instituto Antártico Ecuatoriano por el apoyo brindado para la realización de esta investigación. 


\section{Referencias bibliografícas}

Aguinaco V. 2008. Captura y almacenamiento del dióxido de carbono, Academia de Ingeniería, A.C. México.

Hansell D., Carlson C. 2001. Marine dissolved organic matter and the carbon cycle; Oceanography. 14(4): 41-49.

Herzog H., Eliasson B., Kaarstad O. 2000. Capturing greenhouse gases; Scientific American. 282(2): 72-79.

Hilbertz W. H. 1979. Electrodeposition of minerals in sea water: Experiments and applications; IEEE Journal on Oceanic Engineering. OE-4(3): 1-19.

Jitaru M. 2007. Electrochemical carbon dioxide reduction - fundamental and applied topics. Journal of the University of Chemical Technology and Metallurgy. 42 (4): 333-344.

Levitan D. 2013. Turning $\mathrm{CO}_{2}$, pumped underground, into stone. Scientific American. 309(6): 33-34

Manahan S. 2007. Introducción a la Química Ambiental, Reverté. México.

Metz B, Davidson O, de Coninck H, Loos M, Meyer L. (ed) 2005. La captación y el almacenamiento de dióxido de carbono. Resumen para responsables de políticas. Grupo Intergubernamental de Expertos sobre el Cambio Climático, OMM, PNUMA.

Pontes M. 2011. Composición del agua de mar (en línea). [Fecha de consulta: 12 de julio de 2012]. Dirección electrónica de M@re Nostrum: http://marenostrum.org/curiosidades/composicion/index.htm 